\title{
Pharmacodynamics simulation of HOEC by a computational model of arachidonic acid metabolic network
}

\author{
Wen Yang ${ }^{1, \dagger}$, Xia Wang ${ }^{1, \dagger}$, Kenan $\mathrm{Li}^{2, \dagger}$, Yuanru Liu ${ }^{1}$, Ying Liu ${ }^{2, \star}$, Rui Wang ${ }^{1, \star}$, Honglin $\mathrm{Li}^{1, *}$ \\ ${ }^{1}$ Shanghai Key Laboratory of New Drug Design, School of Pharmacy, East China University of Science and Technology, \\ Shanghai 200237, China \\ 2 BNLMS, State Key Laboratory for Structural Chemistry of Unstable and Stable Species, College of Chemistry and Molecular \\ Engineering, Peking University, Beijing, China \\ *Correspondence: liuying@pku.edu.cn, ruiwang@ecust.edu.cn, hlli@ecust.edu.cn
}

Received July 6, 2018; Revised September 28, 2018; Accepted September 30, 2018

Background: Arachidonic acid (AA) metabolic network is activated in the most inflammatory related diseases, and small-molecular drugs targeting AA network are increasingly available. However, side effects of above mentioned drugs have always been the biggest obstacle. (+)-2-(1-hydroxyl-4-oxocyclohexyl) ethyl caffeate (HOEC), a natural product acted as an inhibitor of 5-lipoxygenase (5-LOX) and 15-LOX in vitro, exhibited weaker therapeutic effect in high dose than that in low dose to collagen induced arthritis (CIA) rats. In this study, we tried to elucidate the potential regulatory mechanism by using quantitative pharmacology.

Methods: First, we generated an experimental data set by monitoring the dynamics of AA metabolites' concentration in A23187 stimulated and different doses of HOEC co-incubated RAW264.7. Then we constructed a dynamic model of A23187-stimulated AA metabolic model to evaluate how a model-based simulation of AA metabolic data assists to find the most suitable treatment dose by predicting the pharmacodynamics of HOEC.

Results: Compared to the experimental data, the model could simulate the inhibitory effect of HOEC on 5-LOX and 15-LOX, and reproduced the increase of the metabolic flux in the cyclooxygenase (COX) pathway. However, a concomitant, early-stage of stimulation-related decrease of prostaglandins (PGs) production in HOEC incubated RAW264.7 cells was not simulated in the model.

Conclusion: Using the model, we predict that higher dose of HOEC disrupts the flux balance in COX and LOX of the AA network, and increased COX flux can interfere the curative effects of LOX inhibitor on resolution of inflammation which is crucial for the efficient and safe drug design.

Keywords: arachidonic acid; metabolic network; computational model; anti-inflammation; natural product

\begin{abstract}
Author summary: Arachidonic acid network is a complex system with many pathways to which non-steroidal antiinflammatroy drugs (NSAIDs) target. However, side effects have always been the disadvantage of these medicines. Using a natural LOX inhibitor HOEC as probe, we established a computational model to simulate the flux regulation of arachidonic acid network after HOEC treatment. Meanwhile, the experimental data of metabolites of different pathways were used to validate and optimize the model. Our study provides an alternitive in NSAIDs design. In addition, it can also guide clinical medication to avoid side effects.
\end{abstract}

These authors contributed equally to this work. 


\section{INTRODUCTION}

Inflammation is a ubiquitous physiological reaction participating in many kinds of diseases, such as rheumatoid arthritis, atherosclerosis, Alzheimer's disease and cancer [1-3], in which a network of inflammatory mediators plays important roles. When cells were stimulated by the pro-inflammatory factors, membrane phospholipid was catalyzed by cytosolic phospholipase $(\mathrm{cPLA})_{2}$ to release arachidonic acid (AA) which touches off the inflammatory cascade. There are four AA metabolic pathways which are prostaglandins (PGs) and thromboxanes (TXs) catalyzed by cyclooxygenase (COX), leukotrienes (LTs) catalyzed by 5-lipoxygenase (LOX), 15-hydroxy-eicosatetraenoic acid (HETE) or lipoxins (LXs) catalyzed by 15-LOX, 12-HETE or LXs catalyzed by 12 -LOX $[4,5]$.

Among the metabolites of COX pathway, $\mathrm{PGE}_{2}$ is the most important pro-inflammatory mediator in pain [6] and edema [7], and has been reported to have anti-inflammatory effects on activating anti-inflammatory factors [8]. $\mathrm{TXA}_{2}$ plays a pivotal role in platelet aggregation [9] while $\mathrm{PGI}_{2}$ can inhibit the platelet aggregation [10]. $\mathrm{PGD}_{2}$ can be metabolized to $\mathrm{PGF}_{2 a}, \mathrm{PGJ}_{2}$ and $15 \mathrm{~d}-\mathrm{PGJ}_{2}$, and $15 \mathrm{~d}-$ $\mathrm{PGJ}_{2}$ is reported as an anti-inflammatory factor [11]. There are three kinds of lipoxygenases in LOX pathway: 5-LOX, 15-LOX and 12-LOX. Among the metabolites of 5 -LOX, $\mathrm{LTB}_{4}$ is a potent pro-inflammatory mediator with neutrophil chemotaxis and adherence involved in almost all inflammatory reactions [12]. As the metabolites of $\mathrm{LTC}_{4}$, cysteamine leukotrienes (CysLTs) are main proinflammatory mediators in asthma and allergic rhinitis $[13,14]$. Others, 5-HETE and some downstream metabolites have pro-inflammatory effects [15]. 12-HETE and 15-HETE, the main metabolites of 12-LOX and 15-LOX pathway, also act as pro-inflammatory factors. Moreover, 12/15-LOX participates in the synthesis of anti-inflammatory factor LXs [5].

A series of anti-inflammatory drugs targeting AA metabolic pathways have been developed. Nonsteroidal anti-inflammatory drugs (NSAIDs), such as aspirin, ibuprofen and indomethacin are classical COX inhibitors, have been routinely used in the treatment of acute and chronic inflammation. However, long-term medication of NSAIDs usually causes gastrointestinal toxicity and mild bleeding due to COX-1 inhibition [16]. Then, selective COX-2 inhibitors were developed to alleviate above mentioned side effects. However, due to cardiovascular risk, rofecoxib was withdrawn in 2004 [17,18]. Zileuton is the only approved 5-LOX inhibitor which is used in the treatment of asthma [19]. Disappointingly, the development of novel 5-LOX inhibitors is not effective as desired which was also blocked by the side effect [20]. In fact, in the past decades, dual and multi-target inhibitors which can interfere few branches of AA metabolic pathways have been considered as more promising research strategy [21].

Network dynamic model, an assistant tool of drug design and screening, provides a new window for understanding the biological process entirely and is gradually replacing the golden mode of target-oriented drug design used in traditional drug discovery. The intrinsic robustness of living systems against various perturbations is a key factor that target-oriented drug did not work as expected and even had inevitable side effects [22]. AA metabolic pathway is also a metabolic network, and the effect of drugs targeting on single or multi-nodes of this network should be evaluated from the point of network instead of the specific target. Lai et al. have established a dynamic model of AA metabolic network in human polymorphous leukocytes using ordinary differential equations (ODEs) [23] and applied the model to simulate the effects of drugs and developed strategies for multiple-target drug design [24]. However, the model does not involve the selection of dose which is also an important factor affecting the network.

(+)-2-(1-hydroxyl-4-oxocyclohexyl) ethyl caffeate (HOEC) was isolated from Incarvillea mairei var. granditlora (Wehrhahn) Grierson [25]. The plants of the Incarvillea genus have long been used as folk medicines for the treatment of inflammatory related diseases in China. In the pharmaceutical activity research of HOEC, 5-LOX and 15-LOX were found to be inhibited in molecular level [26]. The half inhibitory concentration $\left(\mathrm{IC}_{50}\right)$ of HOEC on 5-LOX and 15-LOX activity are $34.6 \pm 5.7 \mu \mathrm{M}$ and $7.8 \pm 0.8 \mu \mathrm{M}$, respectively. In our previous study, HOEC exhibited weaker therapeutic effect at higher dose than that of lower dose when treating collagen induced arthritis (CIA) rats. This result suggests that over inhibiting one pathway of AA metabolic network can even induce unexpected output. Therefore, the prediction of potential regulatory mechanism is a major challenge in targeted anti-inflammatory therapy.

In this study, A23187 (calcium ionophore) was used to stimulate AA metabolism model in macrophage. Firstly, we constructed the topological structure of AA metabolic network by consulting the literature, and used ODEs to describe the reactions involved in the topological structure. Then, a computational model of AA metabolic network in macrophage was established by solving these ODEs with MATLAB (https://www.mathworks.com/products/matlab.html), and the pharmacodynamics of different doses of HOEC was simulated by using this model. At the same time, the effects of different doses of HOEC on the quantitative changes of key metabolites of AA in A23187-stimulated RAW264.7 were measured by ELISA. Our work provides a computational method for 
predicting effective and safe dosage and explaining unexpected efficacy.

\section{RESULTS}

\section{Dynamic profile of AA metabolism in RAW264.7}

The quantification of AA metabolites was measured by ELISA. As shown in Figure 1A, all AA metabolites in control group were stable during the incubation which could eliminate the interference of experimental condition on the cells. In Figure 1B, AA and its metabolites had obvious changes after stimulated by A23187. AA increased right after A23187 stimulation and reached to a peak at $10 \mathrm{~min}$. After a short decrease, AA showed a second wave of slow but continuous increase from 20 min. The profile of 5-LOX metabolites, $\mathrm{LTB}_{4}$ and $\mathrm{LTC}_{4}$, were similar to that of $\mathrm{AA}$ except for the shift in time points (Figure 1B). And the $\mathrm{LTC}_{4}$ 's profile was consistent with that in the ATP stimulated RAW264.7 [27]. cPLA $_{2}$ mediates AA release and 5-LOX activation following cell stimulation caused by the increase of intracellular $\mathrm{Ca}^{2+}$ [28]. The rise extent of $\mathrm{LTC}_{4}$ was greater than $\mathrm{LTB}_{4}$ in present study. The main reason might be that $\mathrm{LTA}_{4}$ is more apt to synthetize $\mathrm{LTC}_{4}$ in eosinophile granulocyte, basophile granulocyte, mast cell and macrophage which is catalyzed by $\mathrm{LTC}_{4} \mathrm{~S}$, while $\mathrm{LTB}_{4}$ is more likely to be generated in neutrophile granulocyte hydrolyzed by $\mathrm{LTA}_{4} \mathrm{H}$ [29-31]. As for the 15-LOX pathway, 15-HETE rose gently to $10 \mathrm{~min}$ after the stimulation of A23187, then fell until $40 \mathrm{~min}$ and rose again. We noted that the base flux of COX-2 pathway were much higher than that of LOX pathway, though the change extent of 5-LOX metabolites was more obvious (Figure 1B). There was a momentary increase of $\mathrm{PGE}_{2}, \mathrm{TXB}_{2}$ and $\mathrm{PGD}_{2}$ at $2.5 \mathrm{~min}$ with an immediately decline to the baseline level. Similar change tendency was reported in the dynamic generation of $\mathrm{PGE}_{2}$ in A23187-stimulated 3T3 cells [32].

Taken together, cPLA ${ }_{2}$ was activated by A23187, then catalyzed AA release and started up the production of AA metabolites simultaneously. The activation of LOX pathway was durative while the activation of COX pathway was ephemeral. Since AA level gradually enhanced after $20 \mathrm{~min}$, we chose the experimental data of first $20 \mathrm{~min}$ in the AA metabolic network modeling.

After treated with $\mathrm{HOEC}, \mathrm{LTB}_{4}$ and $\mathrm{LTC}_{4}$ were inhibited dose-dependently (Figure 2). $50 \mu \mathrm{M}$ HOEC could completely inhibit the $\mathrm{LTB}_{4}$ production (Figure $2 \mathrm{~A}$ ) while $15 \mu \mathrm{M}$ HOEC could completely inhibit the $\mathrm{LTC}_{4}$ production (Figure 2B). It suggested that HOEC had a stronger inhibitory effect on the production of $\mathrm{LTC}_{4}$ than that of $\mathrm{LTB}_{4}$. Although HOEC has a low $\mathrm{IC}_{50}$ on 15 -LOX in enzyme level, it probably weakly inhibited 15 -LOX in RAW264.7. The production of 15-HETE was dosedependently inhibited by HOEC in the first $20 \mathrm{~min}$ following A23187 stimulation and then increased after 20 min. We speculate this flux increase in 15-LOX pathway is the feedback regulation of the AA metabolic network which is caused by the stronger inhibition of 5-LOX pathway and the increase of total AA after 20 min.

As for the COX pathway, the levels of $\mathrm{PGE}_{2}, \mathrm{TXB}_{2}$ and $\mathrm{PGD}_{2}$ in $50 \mu \mathrm{M}$ HOEC treatment group were the highest, which might indicate that the strong inhibition of LOX caused a compensatory increase of metabolic flux in the COX pathway (Figure 2D-2F). This result suggested that over inhibition on the 5-LOX pathway might cause an incline of metabolic flux to the COX pathway which might explain the non-dose-dependent effect of HOEC in animal arthritis studies. Interestingly, the peak of COX metabolites in $2.5 \mathrm{~min}$ was inhibited by HOEC. A23187 was reported as an activator of p38 mitogen-activated protein kinase (MAPK) [33-35] which could further
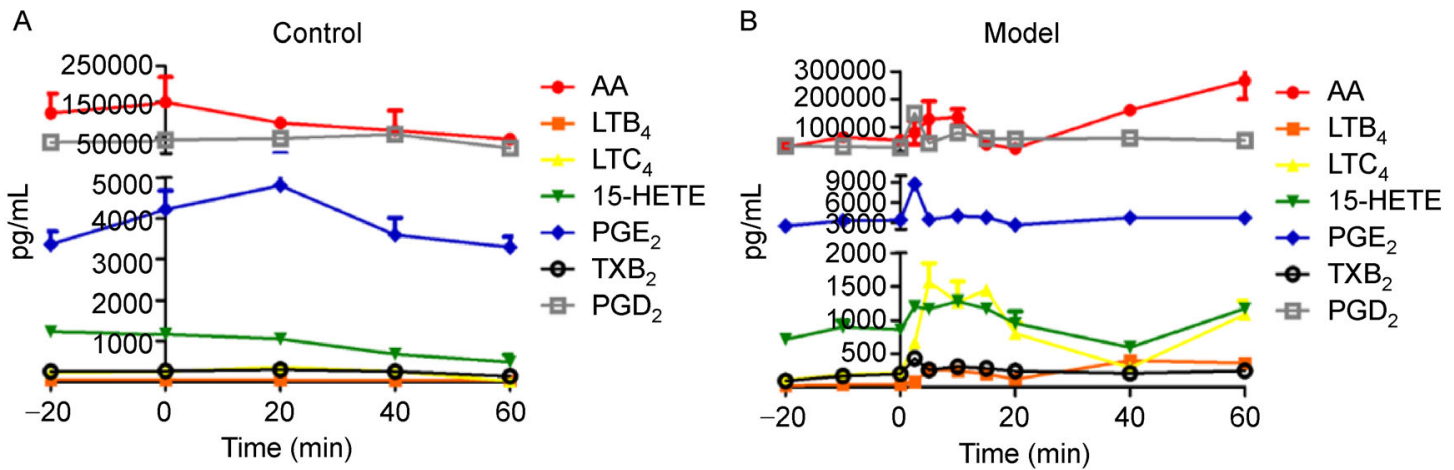

Figure 1. A23187 induced AA metabolites changes in RAW264.7 macrophage. (A) Cells were incubated without stimulation at $37^{\circ} \mathrm{C}$. (B) Cells were incubated at $37^{\circ} \mathrm{C}$ and stimulated by $\mathrm{A} 23187$ at $0 \mathrm{~min}$. The AA metabolites in all samples at each time point were measured by ELISA in triplicate. Data shown are mean \pm SEM. 
A

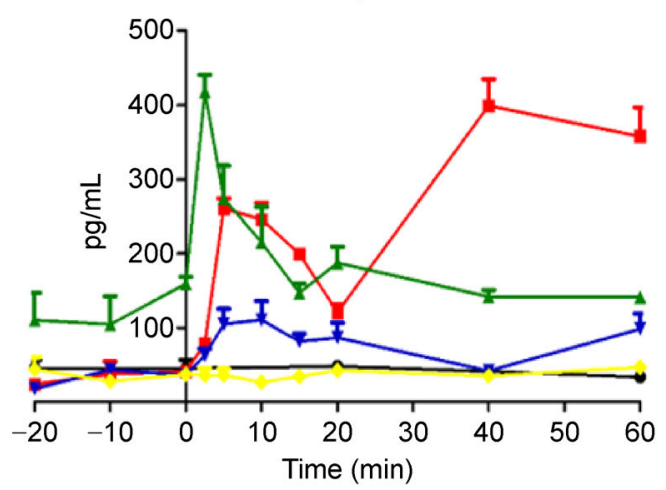

C

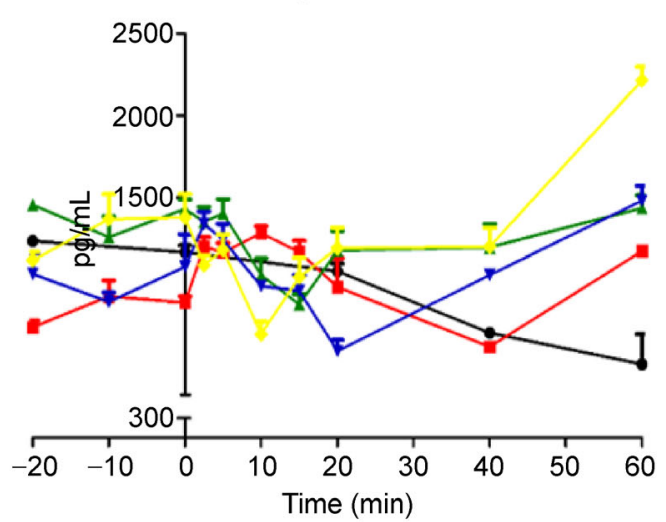

$E$

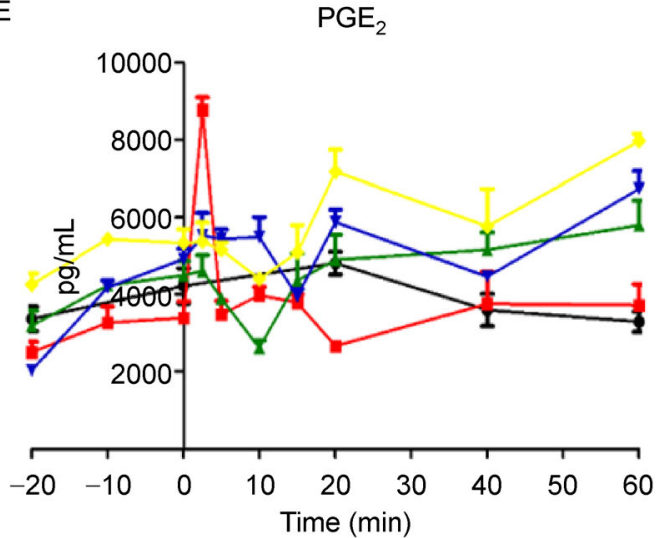

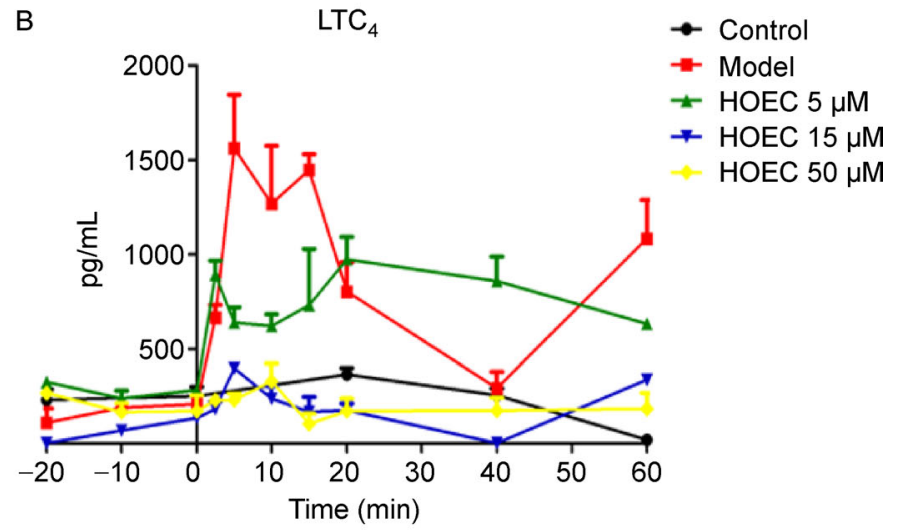

D

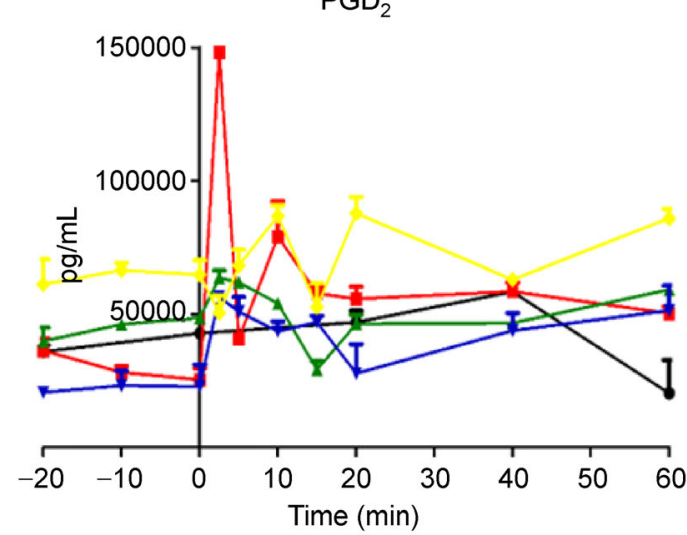

$\mathrm{F}$

$\mathrm{TXB}_{2}$

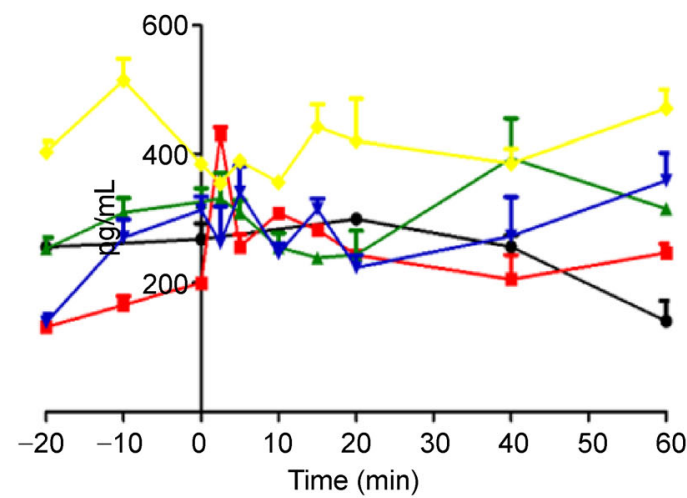

Figure 2. Effect of HOEC on A23187 stimulated flux changes of AA metabolites in RAW264.7 cells. (A-F) Dynamic concentration of $\mathrm{LTB}_{4}, \mathrm{LTC}_{4}, 15-\mathrm{HETE}, \mathrm{PGD}_{2}, \mathrm{PGE}_{2}$ and $\mathrm{TXB}_{2}$. Different concentrations of HOEC were added to HOEC treatment groups, and model was incubated with the same volume of solvent, then $A 23187$ was added (recorded as 0 min). The samples at each time point were harvested, AA metabolites were measured by ELISA in triplicate. Data shown are mean $\pm \mathrm{SEM}$.

affect the phosphorylation and activation of $\mathrm{cPLA}_{2}[33]$. In the previous study of our research group, HOEC was found to inhibit the phosphorylation of p38 MAPK [36]. Therefore, it is possible that HOEC can inhibit the activity of cPLA $_{2}$ by inhibiting the A23187 induced phosphorylation of p38 MAPK, and further inhibit the peak of COX pathway.

\section{Computational model of AA metabolic network in RAW264.7}

Based on KEGG [37], BRENDA [38] and the published dynamic simulation model of AA metabolic network $[23,39]$, a topological structure of AA metabolic network in mouse macrophages was established (Figure 3). As the 


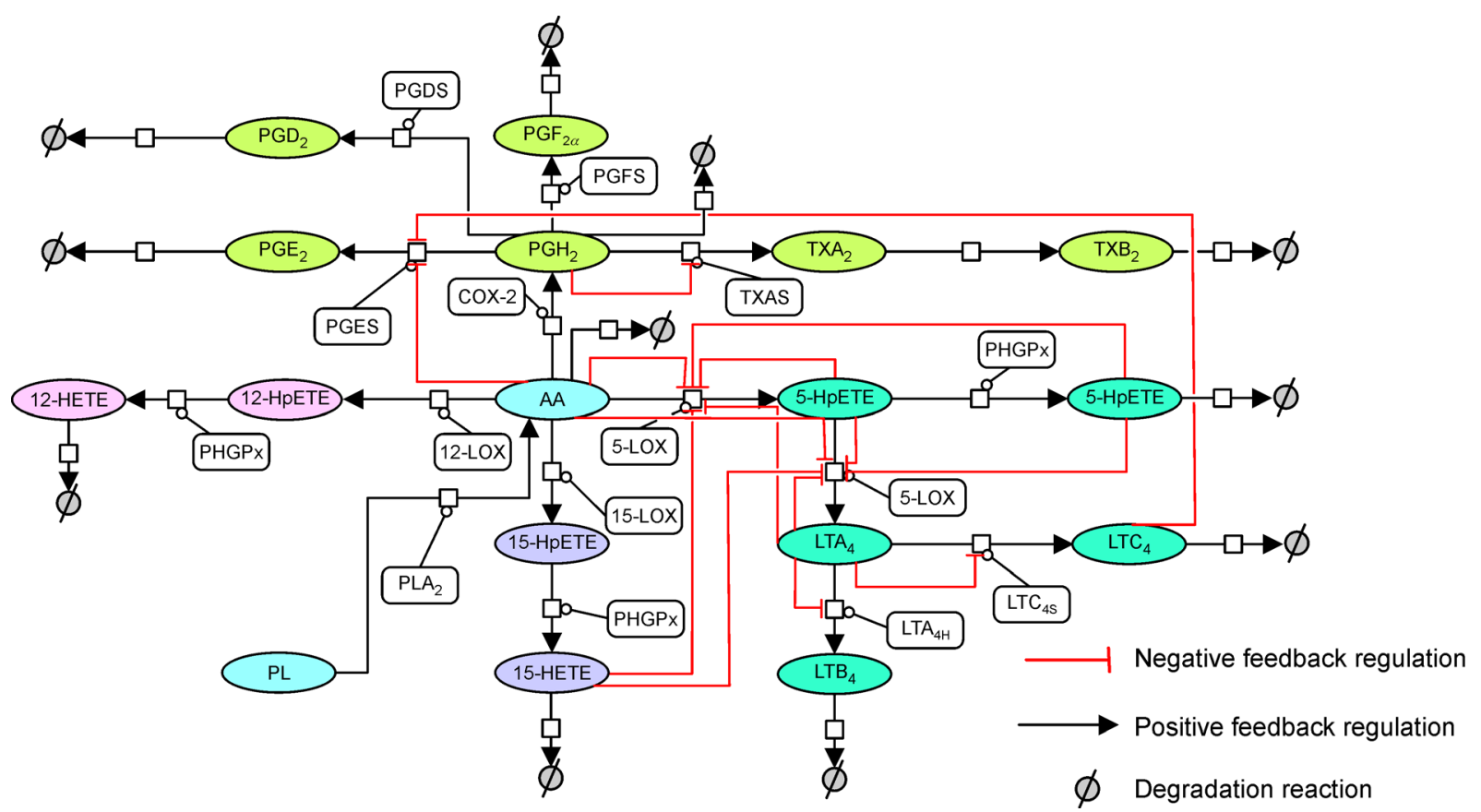

Figure 3. The topological structure of AA metabolic network in mouse macrophages RAW264.7.

figure shown, AA is mainly metabolized by four pathways: the production of PGs via COX pathway; the production of 5-HETE and LTs via 5-LOX pathway; the production of 12-HETE via 12-LOX pathway; the production of 15-HETE via 15-LOX pathway. In order to simplify the model, the non-major metabolites which were not been quantified were excluded. In addition, the further metabolism reactions of AA were directly set as degradation reactions, which were described by the kinetics of first-order chemical reactions.

A series of ODEs were established to simulate unicellular behavior (see details in Materials and Methods), which included 30 initial concentration and 53 reaction parameters (see details in S2.1 and S2.2 of Supplementary Materials). Among the 53 reaction parameters, 32 can be found in the literature. However, these parameters are derived from the results that obtained under different experimental conditions, so further fitting is still needed before they can be used in the final model. After optimization of the parameters, a total of 23 reaction parameters were taken from experimental values, while the others were obtained by fitting the calculated production to experimental data. The parameter set that fit the experimental data well was chosen for the further studies.

After the fitting parameters were confirmed, simulation of the model was carried out. As shown in Figure 4A, the two metabolites of 5-LOX pathway, $\mathrm{LTB}_{4}$ and $\mathrm{LTC}_{4}$, tallied with the experimental values. But the three metabolites of COX pathway did not decrease as rapidly as the experimental values, and the peak value of $\mathrm{PGD}_{2}$ was lower than that of the experimental value. In order to obtain higher content of $\mathrm{PGD}_{2}$, we may have to increase the content of PGDS and $K_{c a t}$ or reduce $K_{m}$ in the model, however, the fitting results based on the above ideas will cause the values of these three parameters to be far beyond the reasonable range. Therefore, the peak fitting of $\mathrm{PGD}_{2}$ is not considered in this model, but its changing trend is regarded as the optimization objective of the model.

Flux analysis was performed on the main pathways in the network, including the 5-LOX, 15-LOX, 12-LOX and $\mathrm{COX}$ pathways. Figure $4 \mathrm{~B}$ shows the profile of metabolites in the first step of AA metabolism. $\mathrm{PGH}_{2}$, 15-HpETE, 5-HpETE, 12-HpETE, the first step metabolites of AA metabolism, were quickly catalyzed by the downstream metabolic enzymes to the more stable metabolites once generated. In addition, the content of $\mathrm{PGH}_{2}$ was the highest among the four metabolites, suggesting that the COX pathway was the main metabolic pathway among the four pathways of AA metabolism, which was consistent with the experimental results. The profile of the downstream metabolites of 5/12/15-LOX pathways is shown in Figure 4C. The results showed that the content of 15-HETE was significantly higher than that of 5-HETE and 12-HETE. As for the downstream metabolites of the 5-LOX pathway, the content of $\mathrm{LTC}_{4}$ was significantly higher than that of $\mathrm{LTB}_{4}, \mathrm{LTA}_{4}$ and 5HETE (Figure 4D). The profile of the four downstream metabolites of the COX pathway is shown in Figure 4E 

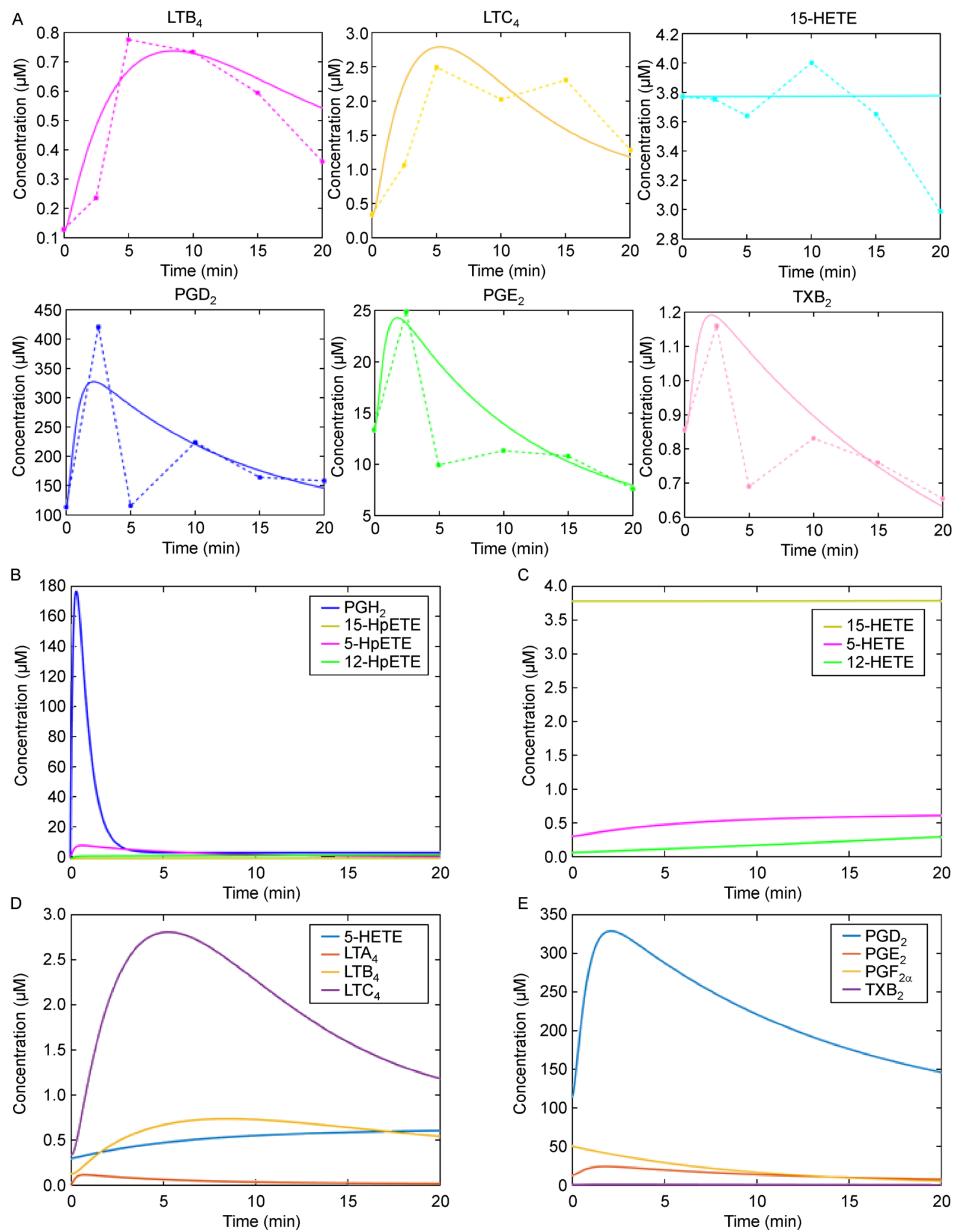

Figure 4. Simulation of model of AA metabolic network in RAW264.7. (A) Comparison of the experimental data and computational results. The calculated results (line: orange represents for $\mathrm{LTB}_{4}$, yellow represents for $\mathrm{LTC}_{4}$, green represents for $\mathrm{PGE}_{2}$, cyan represents for TXB $\mathrm{B}_{2}$, purple represents for $P G D_{2}$, blue represents for 15-HETE) fit with experimental data (dot). (B-E) Flux analysis on the four main pathways: the 5-LOX, 15LOX, 12-LOX and COX pathways. (B) Metabolites in the first step of AA metabolism. (C) Downstream metabolites of 5/12/15-LOX. (D) Downstream metabolites of the 5-LOX pathway. (E) Downstream metabolites of the COX pathway. 
and the content of $\mathrm{PGD}_{2}$ was much higher than that of $\mathrm{PGE}_{2}, \mathrm{PGF}_{2 \mathrm{a}}$ and $\mathrm{TXB}_{2}$, which was consistent with the experimental results.

\section{Pharmacodynamics simulation of HOEC by AA metabolic network model}

Based on the established model of AA metabolic network, the initial concentration of HOEC was set from 0 to 5,15 and $50 \mu \mathrm{M}$, and the simulated results of the changes of metabolites of AA pathway after administration of HOEC was obtained. Take the dose of $50 \mu \mathrm{M}$ as an example, $\mathrm{PGH}_{2}$ was still on a high level, which was slightly higher than that of model group. Due to the inhibitory effect of HOEC on 5-LOX, the content of 5-HpETE, which is the first step metabolite of the 5-LOX pathway, decreased significantly (Figure 5A), but the content of 15-HETE and 12-HETE in the downstream of 15-LOX and 12-HETE pathway did not change significantly compared with the model group (Figure 5B). In addition, all downstream metabolites of 5-LOX pathway were significantly inhibited, and the inhibitory effect on $\mathrm{LTB}_{4}$ and $\mathrm{LTC}_{4}$ was superior to that on 5-HETE (Figure 5C), indicated that $\mathrm{LTA}_{4}$, the precursor of these metabolites, might be from
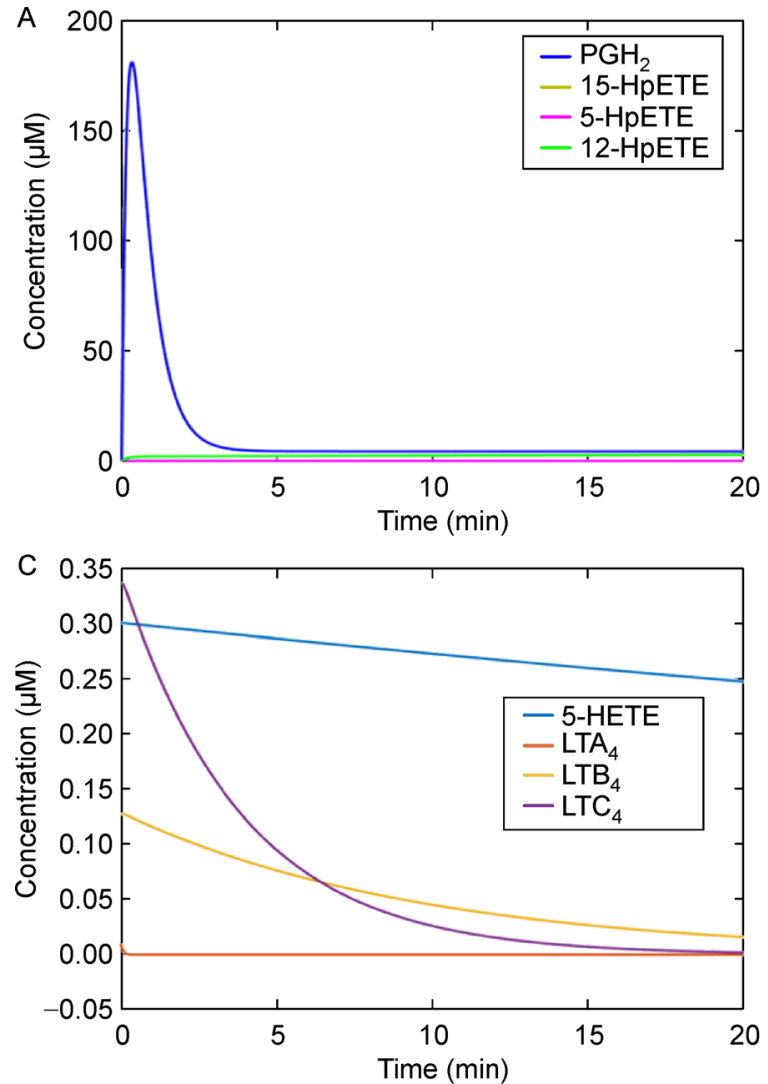

the secondary catalysis of 5-LOX. As shown in Figure $5 \mathrm{D}$, the four metabolites in the downstream of the COX pathway did not have much difference in the change trend and quantitative value compared with the model group. The reason is probably that the flux of 5-LOX pathway is relatively less than COX pathway in whole AA network. Therefore, even if the 5-LOX pathway is completely inhibited, the flux compensation caused by the inhibition of 5-LOX pathway is little on the downstream metabolites of the COX pathway. In addition, the simulation results of the $5 \mu \mathrm{M}$ and $15 \mu \mathrm{M}$ HOEC had similar trends to the $50 \mu \mathrm{M}$, while $50 \mu \mathrm{M}$ HOEC is more effective on the inhibition of 5-LOX pathway and the increase in COX flux. Some unknown parameters of HOEC affecting COX pathway are probably not taken into the model, which resulting in a weak fitting of the simulation results with the experimental data.

\section{DISCUSSION}

Although considerable progress has been made in the genome/proteome-based high-throughput screening methods and rational drug design, the increase in approved drugs in the past decade was not commensurate
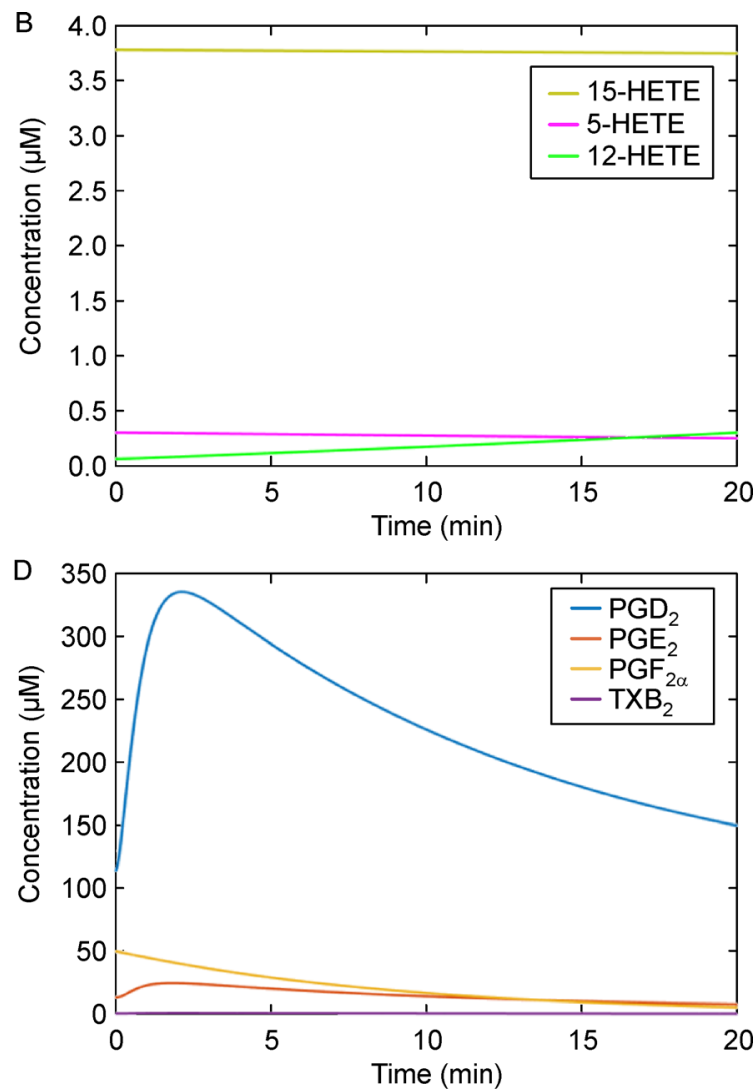

Figure 5. Flux analysis of the four main pathways in the AA metabolic network modulated by HOEC (50 $\boldsymbol{\mu M})$. (A) Metabolites in the first step of AA metabolism. (B) Downstream metabolites of 5/12/15-LOX. (C) Downstream metabolites of the 5-LOX pathway. (D) Downstream metabolites of the COX pathway. 
with the rising cost of drug discovery. Network medicine aims to improve the efficiency of drug development by researching the drug effects and the complexity of diseases at the systematic level. In recent years, application of network medicine has got more and more attention, of which some achievements have been made in infections, cancer, metabolic diseases and neurodegenerative diseases [40]. In this study, HOEC is a natural product with various pharmacological activities which matches the characteristics of multiple targets of traditional Chinese medicine. The above mentioned doseindependent efficacy of HOEC impelled us to explore the regulation of AA metabolic network by HOEC treatment. Using the computational model of AA metabolic network, we simulated the metabolic flux of the network and analyzed the pharmacodynamics of HOEC in macrophages RAW246.7.

The results showed that the model could reproduce the inhibitory effect of HOEC on 5-LOX metabolites and the weak increased flux in COX pathway. However, the model did not simulate the inhibitory effect of HOEC on the peak of COX metabolites at $2.5 \mathrm{~min}$ which indicated that the model was not sound in some direct or indirect effects of HOEC on the COX pathway. Firstly, since cellular membrane is the origin of phospholipid, the substrate is enough to produce AA. So the main factors that determine the production of $\mathrm{AA}$ would be the

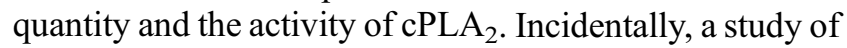
PGs biosynthesis in macrophages [41] provides some ideas for our study. Rossi et al. reported Zileuton, a 5-LOX inhibitor, did not show inhibitory effect on biosynthesis of PGES and COX-2 but resulted in a decrease in AA production by inhibiting the transport of $\mathrm{PLA}_{2}$ to nuclear membrane, and further affected the synthesis of $\mathrm{PGE}_{2}$. Since no further data of biological experiments was provided, we can only make some speculation here: firstly, though HOEC inhibited the instantaneous rise of PGs, metabolites of COX pathway still increased continuously which excluded the possibility of HOEC inhibiting metabolic enzymes in COX pathway. So the inhibition on 5-LOX might induce flux ballooning in other unmeasured pathway which split away the flux peak of COX pathway. Secondly, HOEC might inhibit the p38 MAPK-PLA ${ }_{2}$-COX axis to affect the flux of COX pathway [33-36].

Back to the question introduced in the beginning of the paper, the dose-independent effects of HOEC on arthritis, combined with the results, we speculated that: firstly, the inhibition on the peaks of PGs and TXs in $2.5 \mathrm{~min}$ probably blocked the resolution of inflammation since it has been reported that COX-2 plays an important role in the production of LXs which can give a stop signal to the inflammation $[42,43]$. Secondly, the AA metabolic flux distributed to COX pathway with the increase HOEC.
Though COX-2 can induce the resolution of inflammation, on the other hand, the metabolites of COX pathway are still the major pro-inflammatory mediator in the inflammation [44], so that higher dose of HOEC will decline the anti-inflammatory effect of HOEC. Thirdly, AA can be catalyzed by 15 -LOX to produce 15 -HETE. Some of 15-HETE can be transferred to other cells such as PMNs, and is catalyzed to LXs by 5-LOX [45]. Due to the involvement of 15-LOX and 5-LOX in LXs biosynthesis, high dose of HOEC with more inhibitory effect on 15LOX and 5-LOX might block the anti-inflammatory process. Even 15-HETE is increased at late stage in our cell experiment, the inhibition of 5-LOX can not help the subsequent catalysis of 15-HETE to LXs. Therefore, 15HETE increase is detrimental to the production of LXs. Thus, the experiment results call attention to us that appropriate doses of anti-inflammatory medicines play such an important role to their output of curative effects since they not only work on the targets but also interfere the whole AA metabolic network.

In summary, there are two main limitations of the pharmacodynamics prediction by our AA metabolic network model: firstly, the model was designed only to deal with metabolic enzymes, metabolites and feedback loops of internal AA pathway but no factors of external pathway, such as cytokines [46], protein kinases [47] and signal transduction [48], etc.; secondly, HOEC is multitargeted and some mechanisms of drug action are still not clear, which might limit the accuracy of prediction. The above two factors balance each other: if we have a more comprehensive network model and experimental data of drug effects, we can predict the action targets of drug; or if the target information is comprehensive, we can predict the drug effects accurately through the model. Improvement in both sides can increase efficiency of drug screening, such as chip technology [49,50] and high throughput screen [51,52].

However, it is obvious that there is still a big gap between the pharmacodynamics that predicted by cell model and the actual efficacy in arthritis animal model. In the traditional drug development, there are also many candidate drugs that work in cells or animal models do fail or have different effects on the human level, because the connection of cells with system has always been treated as a black box [53], and the multi-scale model is to solve this problem. A multiscale model is an integrated model that account for more than one level of resolution across measurable domains of time, space, and/or function [54]. At present, the establishment of computational models of inflammation in a single cell environment has a number of application cases [55-58], but the application of multiscale model is rare [59]. In fact, no comprehensive geneto-organism multiscale model has been developed. To work in this direction, we have studied the AA 
metabolites' time-quantitative changes in the CIA rats (unpublished), but the modeling of network in animal model is difficult due to the complexity of systems. With the improvement of modeling method and biological technology, it is believed that the challenging opportunity of multi-scale model can be realized in the near future.

\section{MATERIALS AND METHODS}

\section{Cell culture and collection}

RAW264.7, Abelson murine leukemia virus transformed macrophage (purchased from Chinese Academy of Sciences, Shanghai, China) was cultured with DMEM contained $10 \%$ FBS. Cell passage and amplification every other day with a passage ratio of 4:1 until the sufficient amount of cells for experiment were obtained. Cells were digested by $0.25 \%$ pancreatic enzymes contained $0.02 \%$ EDTA, then centrifuged and suspended in DMEM with a concentration of $10^{7}$ cells $/ \mathrm{mL}$ for experiment.

\section{Macrophage AA metabolism model and HOEC treatment}

$1 \mathrm{~mL}$ of cell suspension was pipetted into each of polypropylene tubes and putted into constant temperature metal bath $\left(37^{\circ} \mathrm{C}, 300 \mathrm{rpm}\right)$. There were five groups: control, model, HOEC $5 \mu \mathrm{M}$, HOEC $15 \mu \mathrm{M}$ and HOEC $50 \mu \mathrm{M}$; 5 time points for control: $-20,0,20,40,60 \mathrm{~min}$; 10 time points for other groups: $-20,-10,0,2.5,5,10$, $15,20,40,60 \mathrm{~min} .10 \mu \mathrm{L} 100 \times$ solution of above three doses of HOEC was added into each tube successively, and equal amounts of solvent was added to the control and model groups, then incubated for $20 \mathrm{~min} .0 \mathrm{~min}$ is the start of A23187 stimulation, $10 \mu \mathrm{L} 100 \times$ solution of $20 \mu \mathrm{M}$ A23187 was added to the model and HOEC treatment group, and equal amounts of solvent was added to the control group. To stop the reaction on the preset time point, three times volume of ice alcohol was added to the cell suspension and the mixture was shaken thoroughly to extract the substance being examined.

\section{Measurement of eicosanoids by ELISA}

The mixture obtained in last chapter was centrifuged with $3000 \times \mathrm{g}$ for $15 \mathrm{~min}$ at $4^{\circ} \mathrm{C}$. Transferred the supernatant to a clean polypropylene tube. Evaporated the ethanol completely under a gentle stream of nitrogen at room temperature. The sample was resuspended in $250 \mu \mathrm{L}$ EIA buffer, then vortexed. The concentrations of AA, $\mathrm{LTB}_{4}$, $\mathrm{LTC}_{4}, 15$-HETE, $\mathrm{PGE}_{2}, \mathrm{TXB}_{2}, \mathrm{PGD}_{2}$ were measured using ELISA kit (Mlbio, China, and Cayman chemical, USA). The assay was performed triplicated according to the manufacturer's instructions.

\section{Simulation methods of mathematical network model}

Based on the kinetic principle of chemical reaction, an ODE is established for each reaction to characterize its reaction rate (see details in S1 of Supplementary Materials). Michaelis-Mentan equations are used to describe the enzymatic reactions in the network:

$$
\frac{\mathrm{d}[S]}{\mathrm{d} t}=\frac{K_{c a t}\left[E_{t}\right][S]}{K_{m}+[S]},
$$

where $K_{c a t}$ is the turnover number, $K_{m}$ is the MichaelisMentan constant, $[S]$ is the substrate concentration, and $\left[E_{t}\right]$ is the total concentration of the enzyme.

If competitive reversible inhibitors exist in the reaction, the kinetic relationship between inhibitors, substrates and reaction rates is:

$$
\frac{\mathrm{d}[S]}{\mathrm{d} t}=\frac{K_{c a t}\left[E_{t}\right][S]}{K_{m}\left(1+\frac{[I]}{K_{i}}\right)+[S]},
$$

where $K_{i}$ is the inhibition constant and $[I]$ is the concentration of inhibitor.

If there are activators that can up-regulate the catalytic reaction, the kinetic relationship between activator, substrate and reaction rate is:

$$
\frac{\mathrm{d}[S]}{\mathrm{d} t}=\frac{K_{c a t}\left(1+\frac{[U]}{K I}\right)\left[E_{t}\right][S]}{K_{m}+[S]},
$$

where $K I$ is the activation constant and $[U]$ is the concentration of activator.

If there are irreversible inhibitors in the reaction, we assume that the enzyme concentration will attenuate according to the following equation:

$$
\frac{\mathrm{d}[E]}{\mathrm{d} t}=-K_{i}[E][I] .
$$

The reaction rate of the drug molecules in the model is:

$$
[I]=-k_{o n} \times[E][I]+k_{o f f}[E I],
$$

where the binding rate constant and dissociation rate constant of the drug molecule with its target are described by $K_{\text {off }}$ and $K_{o n}$, respectively. The relationship between the two is:

$$
K_{i}=\frac{[E][I]}{[E I]}=\frac{k_{o f f}}{k_{o n}} .
$$

The SBPDgui toolkit of Matlab 7.0 was used to solve these ODEs. The metabolic curves of metabolites in AA metabolic network were calculated. These calculated curves were fit to the experimental data by modulating 
parameters that had no direct values from published experiments, while the other parameters remained fixed to their experimental values [23]. In the parameter modulating process, we simulated the model by using the optimization algorithms and the default calculation parameters in the toolkit, and then analyzed the results to determine the reasonable optimization algorithm and calculation conditions. Finally, the optimization values of all the parameters involved in the model and the initial values of the variables were obtained. After the establishment of the model, the AA metabolic network model could be simulated and predicted by using the SBTOOLBOX tool of Matlab 7.0.

\section{SUPPLEMENTARY MATERIALS}

The supplementary materials can be found online with this article at https:// doi.org/10.1007/s40484-018-0163-4.

\section{ACKNOWLEDGEMENTS}

The research is supported in part by the National Key Research and Development Program (No. 2016YFA0502304), Special Program for Applied Research on Super Computation of the NSFC-Guangdong Joint Fund (the second phase, No.U1501501), and the National Natural Science Foundation of China (No. 21173076). Honglin Li is also sponsored by National Program for Special Supports of Eminent Professionals and National Program for Support of Top-notch Young Professionals.

\section{COMPLIANCE WITH ETHICS GUIDELINES}

The authors Wen Yang, Xia Wang, Kenan Li, Yuanru Liu, Ying Liu, Rui Wang and Honglin Li declare that they have no conflict of interests.

All procedures performed in studies were in accordance with the ethical standards of the institution or practice at which the studies were conducted, and with the 1964 Helsinki declaration and its later amendments or comparable ethical standards.

\section{REFERENCES}

1. Libby, P. (2007) Inflammatory mechanisms: the molecular basis of inflammation and disease. Nutr. Rev., 65, S140-S146

2. Davies, P., Bailey, P. J., Goldenberg, M. M. and Ford-Hutchinson, A. W. (1984) The role of arachidonic acid oxygenation products in pain and inflammation. Annu. Rev. Immunol., 2, 335-357

3. Marx, J. (2004) Cancer research: inflammation and cancer: the link grows stronger. Science, 306, 966-968

4. Needleman, P., Truk, J., Jakschik, B. A., Morrison, A. R. and Lefkowith, J. B. (1986) Arachidonic acid metabolism. Annu. Rev. Biochem., 55, 69-102

5. Kühn, H. and O’Donnell, V. B. (2006) Inflammation and immune regulation by 12/15-lipoxygenases. Prog. Lipid Res., 45, 334-356

6. Harvey, R. J., Depner, U. B., Wässle, H., Ahmadi, S., Heindl, C., Reinold, H., Smart, T. G., Harvey, K., Schütz, B., Abo-Salem, O. M., et al. (2004) GlyR $\alpha 3$ : an essential target for spinal $\mathrm{PGE}_{2}-$ mediated inflammatory pain sensitization. Science, 304, 884-887

7. Guay, J., Bateman, K., Gordon, R., Mancini, J. and Riendeau, D. (2004) Carrageenan-induced paw edema in rat elicits a predominant prostaglandin $\mathrm{E}_{2}\left(\mathrm{PGE}_{2}\right)$ response in the central nervous system associated with the induction of microsomal $\mathrm{PGE}_{2}$ synthase-1. J. Biol. Chem., 279, 24866-24872

8. Nakanishi, M. and Rosenberg, D. W. (2013) Multifaceted roles of $\mathrm{PGE}_{2}$ in inflammation and cancer. Semin. Immunopathol., 35, 123-137

9. Smith, J. B., Araki, H. and Lefer, A. M. (1980) Thromboxane $\mathrm{A}_{2}$, prostacyclin and aspirin: effects on vascular tone and platelet aggregation. Circulation, 62, V19-V25

10. Honn, K. V., Cicone, B. and Skoff, A. (1981) Prostacyclin: a potent antimetastatic agent. Science, 212, 1270-1272

11. Scher, J. U. and Pillinger, M. H. (2005) 15d-PGJ2: the antiinflammatory prostaglandin? Clin. Immunol., 114, 100-109

12. Palmblad, J., Malmsten, C. L., Udén, A. M., Rådmark, O., Engstedt, L. and Samuelsson, B. (1981) Leukotriene B4 is a potent and stereospecific stimulator of neutrophil chemotaxis and adherence. Blood, 58, 658-661

13. Csoma, Z., Kharitonov, S. A., Balint, B., Bush, A., Wilson, N. M. and Barnes, P. J. (2002) Increased leukotrienes in exhaled breath condensate in childhood asthma. Am. J. Respir. Crit. Care Med., $166,1345-1349$

14. Peters-Golden, M., Gleason, M. M. and Togias, A. (2006) Cysteinyl leukotrienes: multi-functional mediators in allergic rhinitis. Clin. Exp. Allergy, 36, 689-703

15. Sozzani, S., Zhou, D., Locati, M., Bernasconi, S., Luini, W., Mantovani, A. and O'Flaherty, J. T. (1996) Stimulating properties of 5-oxo-eicosanoids for human monocytes: synergism with monocyte chemotactic protein-1 and -3. J. Immunol., 157, 46644671

16. Rainsford, K. D. (1999) Profile and mechanisms of gastrointestinal and other side effects of nonsteroidal anti-inflammatory drugs (NSAIDs). Am. J. Med., 107, 27-35

17. Psaty, B. M. and Furberg, C. D. (2005) COX-2 inhibitors-lessons in drug safety. N. Engl. J. Med., 352, 1133-1135

18. Singh, D. (2004) Merck withdraws arthritis drug worldwide. BMJ, $329,816.2$

19. Berger, W., De Chandt, M. T. and Cairns, C. B. (2007) Zileuton: clinical implications of 5-Lipoxygenase inhibition in severe airway disease. Int. J. Clin. Pract., 61, 663-676

20. Pergola, C. and Werz, O. (2010) 5-Lipoxygenase inhibitors: a review of recent developments and patents. Expert Opin. Ther. Pat., 20, 355-375

21. Bertolini, A., Ottani, A. and Sandrini, M. (2001) Dual acting antiinflammatory drugs: a reappraisal. Pharmacol. Res., 44, 437-450

22. Kitano, H. (2007) A robustness-based approach to systemsoriented drug design. Nat. Rev. Drug Discov., 6, 202-210

23. Yang, K., Ma, W., Liang, H., Ouyang, Q., Tang, C. and Lai, L. (2007) Dynamic simulations on the arachidonic acid metabolic network. PLOS Comput. Biol., 3, e55

24. Meng, H., Liu, Y. and Lai, L. (2015) Diverse ways of perturbing the human arachidonic acid metabolic network to control 
inflammation. Acc. Chem. Res., 48, 2242-2250

25. Su, Y. Q., Zhang, W. D., Zhang, C., Liu, R. H. and Shen, Y. H. (2008) A new caffeic ester from Incarvillea mairei var. granditlora (Wehrhahn) Grierson. Chin. Chem. Lett., 19, 829-831

26. Li, L., Zeng, H., Liu, F., Zhang, J., Yue, R., Lu, W., Yuan, X., Dai, W., Yuan, H., Sun, Q., et al. (2012) Target identification and validation of (+)-2-(1-hydroxyl-4-oxocyclohexyl) ethyl caffeate, an anti-inflammatory natural product. Eur. J. Inflamm., 10, $297-$ 309

27. Buczynski, M. W., Stephens, D. L., Bowers-Gentry, R. C., Grkovich, A., Deems, R. A. and Dennis, E. A. (2007) TLR-4 and sustained calcium agonists synergistically produce eicosanoids independent of protein synthesis in RAW264.7 cells. J. Biol. Chem., 282, 22834-22847

28. Leslie, C. C. (2015) Cytosolic phospholipase $\mathrm{A}_{2}$ : physiological function and role in disease. J. Lipid Res., 56, 1386-1402

29. Christmas, P., Weber, B. M., McKee, M., Brown, D. and Soberman, R. J. (2002) Membrane localization and topology of leukotriene C4 synthase. J. Biol. Chem., 277, 28902-28908

30. Funk, C. D. (2001) Prostaglandins and leukotrienes: advances in eicosanoid biology. Science, 294, 1871-1875

31. Honda, Z., Nakamura, M., Miki, I., Minami, M., Watanabe, T., Seyama, Y., Okado, H., Toh, H., Ito, K., Miyamoto, T., et al. (1991) Cloning by functional expression of platelet-activating factor receptor from guinea-pig lung. Nature, 349, 342-346

32. Horton, J. K., Williams, A. S., Smith-Phillips, Z., Martin, R. C. and O'Beirne, G. (1999) Intracellular measurement of prostaglandin E2: effect of anti-inflammatory drugs on cyclooxygenase activity and prostanoid expression. Anal. Biochem., 271, 18-28

33. Kramer, R. M., Roberts, E. F., Um, S. L., Börsch-Haubold, A. G., Watson, S. P., Fisher, M. J. and Jakubowski, J. A. (1996) p38 mitogen-activated protein kinase phosphorylates cytosolic phospholipase $\mathrm{A}_{2}\left(\mathrm{cPLA}_{2}\right)$ in thrombin-stimulated platelets. Evidence that proline-directed phosphorylation is not required for mobilization of arachidonic acid by cPLA 2 . J. Biol. Chem., 271, 2772327729

34. Kozawa, O., Tokuda, H., Matsuno, H. and Uematsu, T. (1999) Involvement of p38 mitogen-activated protein kinase in basic fibroblast growth factor-induced interleukin-6 synthesis in osteoblasts. J. Cell. Biochem., 74, 479-485

35. Tokuda, H., Kozawa, O. and Uematsu, T. (2000) Basic fibroblast growth factor stimulates vascular endothelial growth factor release in osteoblasts: divergent regulation by $\mathrm{p} 42 / \mathrm{p} 44$ mitogen-activated protein kinase and p38 mitogen-activated protein kinase. J. Bone Miner. Res., 15, 2371-2379

36. Shen, J.-N., Xu, L.-X., Shan, L., Zhang, W.-D., Li, H.-L. and Wang, R. (2015) Neuroprotection of (+)-2-(1-hydroxyl-4-oxocyclohexyl) ethyl caffeate against hydrogen peroxide and lipopolysaccharide induced injury via modulating arachidonic acid network and p38-MAPK signaling. Curr. Alzheimer Res., 12, 892-902

37. Kanehisa, M., Goto, S., Hattori, M., Aoki-Kinoshita, K. F., Itoh, M., Kawashima, S., Katayama, T., Araki, M. and Hirakawa, M. (2006) From genomics to chemical genomics: new developments in KEGG. Nucleic Acids Res., 34, D354-D357
38. Schomburg, I., Chang, A., Ebeling, C., Gremse, M., Heldt, C., Huhn, G. and Schomburg, D. (2004) BRENDA, the enzyme database: updates and major new developments. Nucleic Acids Res., 32, D431-D433

39. Yang, K., Bai, H., Ouyang, Q., Lai, L. and Tang, C. (2008) Finding multiple target optimal intervention in disease-related molecular network. Mol. Syst. Biol., 4, 228

40. Csermely, P., Korcsmáros, T., Kiss, H. J., London, G. and Nussinov, R. (2013) Structure and dynamics of molecular networks: a novel paradigm of drug discovery. A comprehensive review. Pharmacol. Ther., 138, 333-408

41. Rossi, A., Pergola, C., Koeberle, A., Hoffmann, M., Dehm, F., Bramanti, P., Cuzzocrea, S., Werz, O. and Sautebin, L. (2010) The 5-lipoxygenase inhibitor, zileuton, suppresses prostaglandin biosynthesis by inhibition of arachidonic acid release in macrophages. Br. J. Pharmacol., 161, 555-570

42. Chan, M. M.-Y., Moore, A. R. (2010) Resolution of inflammation in murine autoimmune arthritis is disrupted by cyclooxygenase-2 inhibition and restored by prostaglandin $\mathrm{E}(2)$-mediated lipoxin A(4) Production. J. Immunol., 184, 6418-6426

43. Rajakariar, R., Yaqoob, M. M. and Gilroy, D. W. (2006) COX-2 in inflammation and resolution. Mol. Interv., 6, 199-207

44. Seibert, K., Zhang, Y., Leahy, K., Hauser, S., Masferrer, J., Perkins, W., Lee, L. and Isakson, P. (1994) Pharmacological and biochemical demonstration of the role of cyclooxygenase 2 in inflammation and pain. Proc. Natl. Acad. Sci. USA, 91, 1201312017

45. Samuelsson, B., Dahlén, S. E., Lindgren, J. A., Rouzer, C. A. and Serhan, C. N. (1987) Leukotrienes and lipoxins: structures, biosynthesis, and biological effects. Science, 237, 1171-1176

46. Dinarello, C. A. (2000) Proinflammatory cytokines. Chest, 118 , 503-508

47. Pettus, B. J., Bielawska, A., Spiegel, S., Roddy, P., Hannun, Y. A and Chalfant, C. E. (2003) Ceramide kinase mediates cytokineand calcium ionophore-induced arachidonic acid release. J. Biol. Chem., 278, 38206-38213

48. Piomelli, D. (1993) Arachidonic acid in cell signaling. Curr. Opin. Cell Biol., 5, 274-280

49. De Micheli, G. and Benini, L. (2006) Networks on Chips: Technology and Tools. Academic Press.

50. Benini, L., De Micheli, G. (2002) Networks on chips: a new SoC paradigm. Computer, 35, 70-78

51. Hopkins, A. L. (2008) Network pharmacology: the next paradigm in drug discovery. Nat. Chem. Biol., 4, 682-690

52. Wang, X., Terfve, C., Rose, J. C. and Markowetz, F. (2011) HTSanalyzeR: an R/Bioconductor package for integrated network analysis of high-throughput screens. Bioinformatics, 27, 879-880

53. Zhao, S. and Iyengar, R. (2012) Systems pharmacology: network analysis to identify multiscale mechanisms of drug action. Annu. Rev. Pharmacol. Toxicol., 52, 505-521

54. Walpole, J., Papin, J. A. and Peirce, S. M. (2013) Multiscale computational models of complex biological systems. Annu. Rev. Biomed. Eng., 15, 137-154

55. Gupta, S., Maurya, M. R., Stephens, D. L., Dennis, E. A. and 
Subramaniam, S. (2009) An integrated model of eicosanoid metabolism and signaling based on lipidomics flux analysis. Biophys. J., 96, 4542-4551

56. Kihara, Y., Gupta, S., Maurya, M. R., Armando, A., Shah, I., Quehenberger, O., Glass, C. K., Dennis, E. A. and Subramaniam, S. (2014) Modeling of eicosanoid fluxes reveals functional coupling between cyclooxygenases and terminal synthases. Biophys. J., 106, 966-975

57. Yang, K., Ma, W., Liang, H., Ouyang, Q., Tang, C. and Lai, L.
(2007) Dynamic simulations on the arachidonic acid metabolic network. PLOS Comput. Biol., 3, e55

58. Yang, K., Bai, H., Ouyang, Q., Lai, L. and Tang, C. (2008) Finding multiple target optimal intervention in disease-related molecular network. Mol. Syst. Biol., 4, 228

59. Fajmut, A., Schäfer, D., Brumen, M., Dobovišek, A., Antić, N. and Emeršič, T. (2015) Dynamic model of eicosanoid production with special reference to non-steroidal anti-inflammatory drugtriggered hypersensitivity. IET Syst. Biol., 9, 204-215 